\title{
Production of human factor VIII-FL in 293T cells using the bicistronic MGMT(P140K)- retroviral vector
}

\author{
A.M. Fontes ${ }^{1,2}$, F.U.F. Melo ${ }^{1}$, L.J. Greene ${ }^{1}$, V.M. Faça ${ }^{1}$, Y. Lin ${ }^{3}$, \\ S.L. Gerson ${ }^{3}$ and D.T. Covas ${ }^{1,2}$ \\ ${ }^{1}$ Hemocentro de Ribeirão Preto, \\ Instituto Nacional de Ciência e Tecnologia em Células Tronco e Terapia Celular, \\ Hospital das Clínicas, Faculdade de Medicina de Ribeirão Preto, \\ Universidade de São Paulo, Ribeirão Preto, SP, Brasil \\ ${ }^{2}$ Departamento de Clínica Médica and Departamento de Genética, \\ Faculdade de Medicina de Ribeirão Preto, Universidade de São Paulo, \\ Ribeirão Preto, SP, Brasil \\ ${ }^{3}$ Case Comprehensive Cancer Center, \\ Case Western Reserve University and Seidman Cancer Center, \\ University Hospitals Case Medical Center, Cleveland, OH, USA \\ Corresponding author: A.M. Fontes \\ E-mail: fontesam@hemocentro.fmrp.usp.br
}

Genet. Mol. Res. 11 (1): 775-789 (2012)

Received February 2, 2012

Accepted March 2, 2012

Published March 22, 2012

DOI http://dx.doi.org/10.4238/2012.March.22.8

\begin{abstract}
Hemophilia A is the most common X-linked bleeding disorder; it is caused by deficiency of coagulation factor VIII (FVIII). Replacement therapy with rFVIII produced from human cell line is a major goal for treating hemophilia patients. We prepared a full-length recombinant FVIII (FVIII-FL), using the pMFG-P140K retroviral vector. The IRES DNA fragment was cloned upstream to the P140K gene, providing a 9.34-kb bicistronic vector. FVIII-FL cDNA was
\end{abstract}


then cloned upstream to IRES, resulting in a $16.6-\mathrm{kb}$ construct. In parallel, an eGFP control vector was generated, resulting in a 10.1$\mathrm{kb}$ construct. The $293 \mathrm{~T}$ cells were transfected with these constructs, generating the 293T-FVIII-FL/P140K and 293T-eGFP/P140K cell lines. In 293T-FVIII-FL/P140K cells, FVIII and P140K mRNAs levels were $4,410( \pm 931.7)$ - and 295,400 $( \pm 75,769)$-fold higher than in virgin cells. In 293T-eGFP/P140K cells, the eGFP and P140K mRNAs levels were 1,501,000 ( $\pm 493,700)$ - and 308,000 ( $\pm 139,300)$-fold higher than in virgin cells. The amount of FVIII-FL was $0.2 \mathrm{IU} / \mathrm{mL}$ and 45 $\mathrm{ng} / \mathrm{mL}$ FVIII cells or $4.4 \mathrm{IU} / \mu \mathrm{g}$ protein. These data demonstrate the efficacy of the bicistronic retroviral vector expressing FVIII-FL and MGMT(P140K), showing that it could be used for producing the FVIIIFL protein in a human cell line.

Key words: Bicistronic retroviral vector; FVIII coagulation factor; MGMT(P140K); eGFP; 293T cells

\section{INTRODUCTION}

Hemophilia A is the most common X-linked bleeding disorder caused by deficiency of coagulation factor VIII (FVIII) with a frequency of one in 5000 males (Furie and Furie, 1988; Soucie et al., 1998). Liver transplantation is the only curative therapy available today (Ashrani et al., 2004; Yokoyama et al., 2011). However, the low rate of organ donations has been the principal problem, and the standard strategy in the care of people with hemophilia A has been replacement therapy with plasma-derived (pdFVIII) and/or recombinant factor VIII (rFVIII) (Manco-Johnson, 2003; Franchini, 2010). Historically, the recombinant era for hemophilia began in the early 1990s, with the approval of the first generation of Chinese hamster ovary (CHO) cell-derived full-length rFVIII (rFVIII-FL) (Recombinate) (Pipe et al., 2008) and baby hamster kidney cell (BHK)-derived rFVIII-FL (Kogenate) (Josephson and Abshire, 2004; Grillberger et al., 2009).

Next, in 2000, after demonstrating that the B domain is not necessary for the procoagulant activity of FVIII (Toole et al., 1986; Fay, 1993), and that B-domain-deleted FVIII (FVIII $\triangle \mathrm{B}$ ) results in a 10- to 20 -fold increase in the levels of mRNA and the primary translation product (Pittman et al., 1993), the second generation of rFVIII named CHO-derived rFVIII $\triangle \mathrm{B}$ (ReFacto) was introduced (Pipe et al., 2008). Also, to reduce the risk of contamination in this second generation, human albumin was used as a nutrient in cell culture but not for stabilizing the final product. However, it was also shown that the B domain plays an important role in the intracellular processing and transport of FVIII and that the FVIII $\Delta \mathrm{B}$ molecule is secreted poorly, due to either reduced folding or inefficient ER-to-Golgi transport (Pipe, 2009). The second generation of BHK-derived rFVIII-FL (Kogenate FS) thereby became commercially available (Pipe, 2008).

In 2003, the third generation came along, which did not contain any animal or human plasma in the culture medium or as a stabilizer in the final product. There were three products: Helixate NexGen (BHK21-derived rFVIII-FL), Advate (CHO-derived rFVIII-FL) and ReFacto (CHO-derived rFVIII $\triangle \mathrm{B}$ ). However, all these products were generated in nonhuman 
mammalian cell lines, which enable the production of recombinant proteins with murine glycosylation pattern. Nowadays, the primary concern of replacement therapy is the development of inhibitors, which may be influenced by either genetic background or nonhuman glycosylation pattern (Goudemand et al., 2006; Van Damme et al., 2006). Immunogenicity not only remains a serious cause of morbidity in hemophilia patients but increases the annual cost for treatment 4-fold (Gringeri et al., 2003). Therefore, an alternative approach should be production using a human cell line.

In 2010, Octapharma started a clinical trial in the US and Germany for treatment of hemophilia A patients using the fourth generation of $\mathrm{rFVIII}$, which is a human embryonic cell line (HEK 293F)-derived rFVIII-DB with a human glycosylation pattern (Human-cl rhFVIII) (http://www.bioportfolio.com/news/article/236108/Octapharma-Clinical-Trial-BeginsIn-The-U-s-And-Germany-Treating-Hemophilia.htm). However, based on previous evidence that portions of the B domain may have functional effects throughout the life cycle of the FVIII molecule (Bovenschen et al., 2005; Pipe, 2009), a number of research groups have generated transgenic cell lines with full-length FVIII (FVIII-FL) (Becker et al., 2004; Pipe et al., 2011).

Moreover, the gene-based approach to hemophilia A using viral vectors has been in development for more than 15 years and offers the possibility to eliminate the burden of protein-replacement therapy. However, up to now, clinical trials using gene therapy for hemophilia A have not been successful (Camire, 2010). On the other hand, pre-clinical studies have provided evidence for the feasibility of using a bicistronic vector expressing the $\gamma$-globin or FIX gene and the $\mathrm{O}(6)$-methylguanine-DNA-methyltransferase (MGMT) gene for in vivo enrichment of genetically modified autologous or allogeneic stem cells for the treatment of $\beta$-thalassemia or hemophilia B mouse models (Persons et al., 2003; Chang et al., 2008; Zhao et al., 2009).

On the basis of these considerations, we constructed a bicistronic retroviral vector containing the MGMT(P140K) gene for treatment of hemophilia A. To determine if MGMTretroviral vector is a suitable expression vector system we constructed two types of expression vectors, one with FVIII-FL and one with the enhanced green fluorescent protein (eGFP). This report describes the use of these vectors for the delivery of biologically active recombinant FVIII-FL or eGFP in human cell lines. We showed that this system can support the maturation of desired proteins and could be a useful platform for the production of recombinant proteins for research and therapeutic purposes.

\section{MATERIAL AND METHODS}

The chemicals used were of molecular biology quality. Platinum Pfx polymerase, TOPO-TA cloning and Escherichia coli (DH5 $\alpha$ ) competent cells were purchased from Invitrogen (USA). dNTPs and DNA size markers were also from Invitrogen. Restriction endonucleases were from New England Biolabs (USA). T4 DNA ligase was from Roche Applied Science (USA). Agarose gel purification, PCR product clean-up kit and plasmid mini-prep kits were all products of Qiagen (USA). We designed the primers using the Oligo Explorer software (version 1.5) on the basis of data in NCBI Gene Sequence. All oligonucleotides were synthesized by Sigma (USA) and their sequences are listed in Table 1. 
Table 1. Sequence of primes used and the sizes of their PCR amplification products.

\begin{tabular}{|c|c|c|c|}
\hline Primer & Primer sequence & Objective & Product size (bp) \\
\hline P5IRESclon & 5'-CTCCCGCTCGTTAACTCGAGGATCCACTAG-3' & Amplification IRES by PCR & 626 \\
\hline P3IRESclon & 5'-CTCTACGTAATTATCATCGTGTTTTTCAAAGGA-3' & & \\
\hline P5IRESMFG & 5'-TTGGATAGTTGTGGAAAGAGTC-3' & Colony IRES screening & 521 \\
\hline P3MFGP140K & 5'-GAAAACGGGATGGTGAAGCG-3' & & \\
\hline P5MFG-FVIIIsa & 5'-CCTCATCTCCAGCAGTCAAG-3' & Colony FVIII screening & 398 \\
\hline P3MFG-IRES & 5'-ACTGCTTCCTTCACGACATT-3' & & \\
\hline P5MFG-F8 1655 & 5'-GCAGCCTACCAAGAACAACT-3' & Sequencing FVIII N-terminal & 524 \\
\hline P3FVIII 2179 & 5'-CCACTGTATCATAAACCTCA-3' & & \\
\hline P5MFG-FVIII 8834 & 5'-CTCGCTACCTTCGAATTCAC-3' & Sequencing FVIII C-terminal & 421 \\
\hline P3MFG-P140K 9265 & 5'-ACATATAGACAAACGCACACC-3' & & \\
\hline P5GFPkh & 5'-CTCGTTAACCGCCACCATGGTGAGCAAGG-3' & Amplification GFP by PCR & 810 \\
\hline P3GFPh & 5'-CTCGTTAACTTATCTAGATCCGGA-3' & & \\
\hline P5MFGeGFP & 5'-AACTACAACAGCCACAACGTC-3' & Colony GFP screening & 643 \\
\hline P3MFGIRESGFP & 5'-TCTTCAAGAAGCTTCCAGAGG-3' & & \\
\hline PFVIII (Taqman) & Hs 00252034-M1 & FVIII amplicon & Not informed \\
\hline P5MGMTH & 5'-CAGTGGGAGGAGCAATGAGA -3' & P140K amplicon & 120 \\
\hline P3MGMTH & 5'-AGAAGCCATTCCTTCACGGC-3' & & \\
\hline P5GFP & 5'-AGCAGAAGAACGGCATCAAGG-3' & eGFP amplicon & 114 \\
\hline P3GFP & 5'-TGGTTGTCGGGCAGCA-3' & & \\
\hline P5GAPDH & 5'-GCCTCAAGATCATCAGCAATGC-3' & GAPDH amplicon & 170 \\
\hline P3GAPDH & 5'-CATGGACTGTGGTCATGAGTCCT-3' & & \\
\hline PGAPDH (Taqman) & & GAPDH amplicon & Not informed \\
\hline
\end{tabular}

IRES = internal ribosomal entry site; GFP = green fluorescent protein; eGFP = enhanced green fluorescent protein; GAPDH $=$ glyceraldehyde 3-phosphate dehydrogenase.

\section{Molecular biology techniques}

Transformation of chemically competent $E$. coli $\mathrm{DH} 5 \alpha$ cells by the heat shock method was based on a method previously described (Cohen et al., 1972).

\section{Retroviral vector construction}

Expression vectors used in the present study were derived from pMFG-P140K. Transgene expression was driven by the MLV 5'LTR promoter. Vector generation was as follows:

- $\quad$ MMFG-I-P140K indicates the pMFG-P140K plus internal ribosomal entry site (IRES) sequence upstream of P140K. To develop this vector, the IRES sequence was PCR-amplified from pLXIN (Clontech, USA) using primers listed in Table 1. PCR amplification was performed with a 2-min pre-heat followed by 30 cycles of DNA denaturation at $94^{\circ} \mathrm{C}$ for $30 \mathrm{~s}$, annealing at $60^{\circ} \mathrm{C}$ for 30 $\mathrm{s}$, and polymerization at $72^{\circ} \mathrm{C}$ for 2 min followed by a 5 -min final extension at $72^{\circ} \mathrm{C}$. The resulting PCR product was purified using gels, restriction digested with $B s r$ BI and SnaBI and ligated into pMFG-MGMT(P140K) after PmlI digestion. Twenty-four colonies were randomly picked and identified using standard colony PCR. Orientation of the positive PCR clones were confirmed by NcoI and $X h o I$ digestion and sequencing. Clone A7 was utilized for subsequent subcloning efforts.

- $\quad$ MMFG-FVIII-FL-I-P140K indicates pMFG-I-P140K plus FVIII-FL upstream of IRES. To develop this vector, the FVIII-FL cDNA, which contains the entire 
B domain was derived from the plasmid pSP64-VIII (ATCC No. 39812). The cDNA was isolated as SalI fragment, and the cohesive ends were completed by DNA polymerase I (Klenow fragment) and blunt-ligated with the XhoI digested plasmid pMFG-I-P140K(A7). It was also completed by DNA polymerase I (Klenow fragment). A total of 24 grown colonies were randomly picked and identified with standard colony PCR. Orientations of the positive PCR clones were confirmed by $N c o$ I and NsiI digestion and sequencing. Clone A6 was utilized for further assays.

- $\quad$ MMFG-eGFP-I-P140K indicates pMFG-I-P140K plus eGFP upstream of IRES. To develop this vector, the eGFP cDNA was PCR-amplified from peGFP-C1 (Clontech, Cat. No. 6084-1) using primers listed in Table 1. PCR amplification was performed as described for IRES amplification, but with the annealing temperature of $66^{\circ} \mathrm{C}$. The resulting PCR product was purified by gel electrophoresis, digested with $B c l \mathrm{I}$ and $H p a \mathrm{I}$, and the cohesive ends were completed by DNA polymerase I (Klenow fragment) and blunt-ligated with the HpaI and BamHI digested plasmid pMFG-I-P140K(A7). Twenty-eight colonies were randomly picked and identified using standard colony PCR. The orientation of the positive PCR clones was confirmed by $N c o$ I digestion and by sequencing. Clone A8 was utilized for further assays.

\section{Cell culture and gene transfer}

Human embryonic kidney cells, 293T (ATCC: CRL 1573) were routinely maintained in Dulbecco's modified Eagle's medium (Life Technologies, USA) supplemented with $10 \%$ fetal bovine serum at $37^{\circ} \mathrm{C}$ in a humidified atmosphere of $5 \% \mathrm{CO}_{2}$. Cells were grown overnight to $50-60 \%$ confluence on $100-\mathrm{mm}^{2}$ plates. Transient transfections were conducted using the lipofectamine method as described elsewhere (Dalby et al., 2004). In brief, the cells were transfected for 20 min with $24 \mu \mathrm{g}$ FVIII-FL plasmid or eGFP plasmid. Approximately $7 \mathrm{~h}$ after transfection, the samples were added to the culture medium. The cells were harvested $72 \mathrm{~h}$ later and assayed for FVIII expression and activity or eGFP expression and fluorescence.

\section{RNA analysis}

Total RNA from retrovirally transfected and nontransfected 293T cells was isolated using the RNeasy Mini-kit (Qiagen, Germany) according to manufacturer instructions. RNA concentration and homogeneity was determined spectrophotometrically by measuring absorbance at 260 and $280 \mathrm{~nm}$. RNA $(2 \mu \mathrm{g})$ was reverse transcribed to cDNA using High-Capacity cDNA Archive kit (Applied Biosystems, USA), according to manufacturer instructions. cDNA $(2 \mu \mathrm{L}$ cDNA diluted $1: 20)$ was applied in the real-time PCR assay to determine the expression of FVIII-FL, eGFP and MGMT(P140K). Gene expression of eGFP and MGMT(P140K) was quantified using the SYBR Green PCR Master Mix kit (Applied Biosystems) and specific primers (Invitrogen Life Technologies) listed in Table 1 on the 7500 Real-Time PCR System (Applied Biosystems). For FVIII expression, TaqMan Universal Master Mix (Applied Biosystems) was used. Cycling was carried out for 2 min at $50^{\circ} \mathrm{C}$, 
followed by denaturation at $95^{\circ} \mathrm{C}$ for $10 \mathrm{~min}$. Amplification was carried out with 40 cycles of $95^{\circ} \mathrm{C}$ for $15 \mathrm{~s}$ and at $60^{\circ} \mathrm{C}$ for $60 \mathrm{~s}$. The specificity of each primer pair was confirmed by melting curve analysis. The GAPDH gene was used as an endogenous control (housekeeping gene). All reactions were carried out in duplicate, and the relative expression levels were calculated using the $2^{\Delta \Delta \mathrm{Ct}}$ method (Pfaffl, 2001) with the mean of the virgin cells used as reference.

\section{FACS analysis and fluorescence microscopy of eGFP expression}

At $72 \mathrm{~h}$ after transfections, about 20,000 events were analyzed by FACS using the CellQuest software (Becton Dickinson) to obtain the percentage of eGFP-positive cells. Four independent experiments were performed. Analysis of 293T culture plates was performed using a digital fluorescence camera and a fluorescence microscope IX71 (Olympus, Japan) with a filter unit for low magnification.

\section{Quantification of FVIII-FL}

293T-FVIII-FL/P140K culture supernatants were filtered $(0.2 \mu \mathrm{m})$ and stored at $-80^{\circ} \mathrm{C}$ before FVIII activity was measured. Before assay, supernatants were concentrated 5- to 10-fold using Amicon Centricon YM-10 centrifugal filter devices with 10-kDa cut-off membranes (Millipore, USA).

Coagulation FVIII activity (FVIII:C) was measured using a Coamatix chromogenic assay (DiaPharma Group, Inc., USA) according to manufacturer instructions. Briefly, the released FVIIIa acts as a cofactor of factor IXa to generate factor Xa. Since FVIIIa is a cofactor to FIXa, it stimulates the reaction. By using optimal amounts of $\mathrm{Ca}^{2+}$, phospholipid, and FIXa, and an excess of FX, the rate of activation of FX is linearly related to the amount of FVIII. FXa hydrolyzes the chromogenic substrate S-2765, releasing the chromophoric group pNA. Absorbance was read at $405 \mathrm{~nm}$, and the generated FXa and amount of absorbance was proportional to the FVIII activity in the sample. The standard curve was obtained with S.A.R.P. (Speciality Assayed Reference Plasma; Australia). Samples and standards were diluted 80-fold with buffer supplied in the kit. FVIII activity in cell culture medium was related to FVIII activity in plasma standard, which is defined as $1 \mathrm{IU}$ hFVIII or $200 \mathrm{ng} / \mathrm{mL}$.

The amount of FVIII antigen (VIIIC:Ag) in the 293T-FVIII-FL/P140K supernatant was measured using the ELISA immunoassay kit Asserachrom VIIIC:Ag (Diagnostica Stago, USA). The assay was performed using a microplate coated with a specific anti-hFVIII antibody, provided in the kit. A standard curve was prepared with 2-fold serial dilutions of the standard lyophilized human normal pooled plasma, containing $200 \mathrm{ng} / \mathrm{mL}$ FVIII, which corresponds to $1 \mathrm{IU} / \mathrm{mL}$. Briefly, FVIII supernatant and plasma reference were diluted 1:4 with the solution supplied ( $40 \mu \mathrm{L}$ sample or control and $120 \mu \mathrm{L}$ buffer) and incubated in precoated plates in duplicate. Control fresh plasma (obtained from Hemocenter Blood Bank) and S.A.R.P. commercial reference plasma were also used as controls. Absorbance was measured at $405 \mathrm{~nm}$. Both the activity and quantification measurements were performed on a plate reader (Model M5, Molecular Devices, USA) and data processed by the SoftMax ${ }^{\circledR}$ Pro Data Acquisition \& Analysis Software. 


\section{RESULTS}

\section{Construction of the pMFG-FVIII-FL-IRES-P140K and pMFG-eGFP-IRES- P140K retroviral plasmids}

\section{PCR cloning of IRES}

To generate the retroviral bicistronic vector pMFG-IRES-P140K $(9.3 \mathrm{~kb})$, the IRES was cloned into pMFG-P140K. Of the 24 colonies, 7 yielded a strong 521-bp PCR product (Figure 1A) with the correct orientation. After colony screening, three positive clones were selected. Analysis by restriction enzyme (Figure 1B) and DNA sequencing (data not shown) confirmed the identity of the construct. The A7 clone denoted pMFG-I-P140K was selected for further studies.

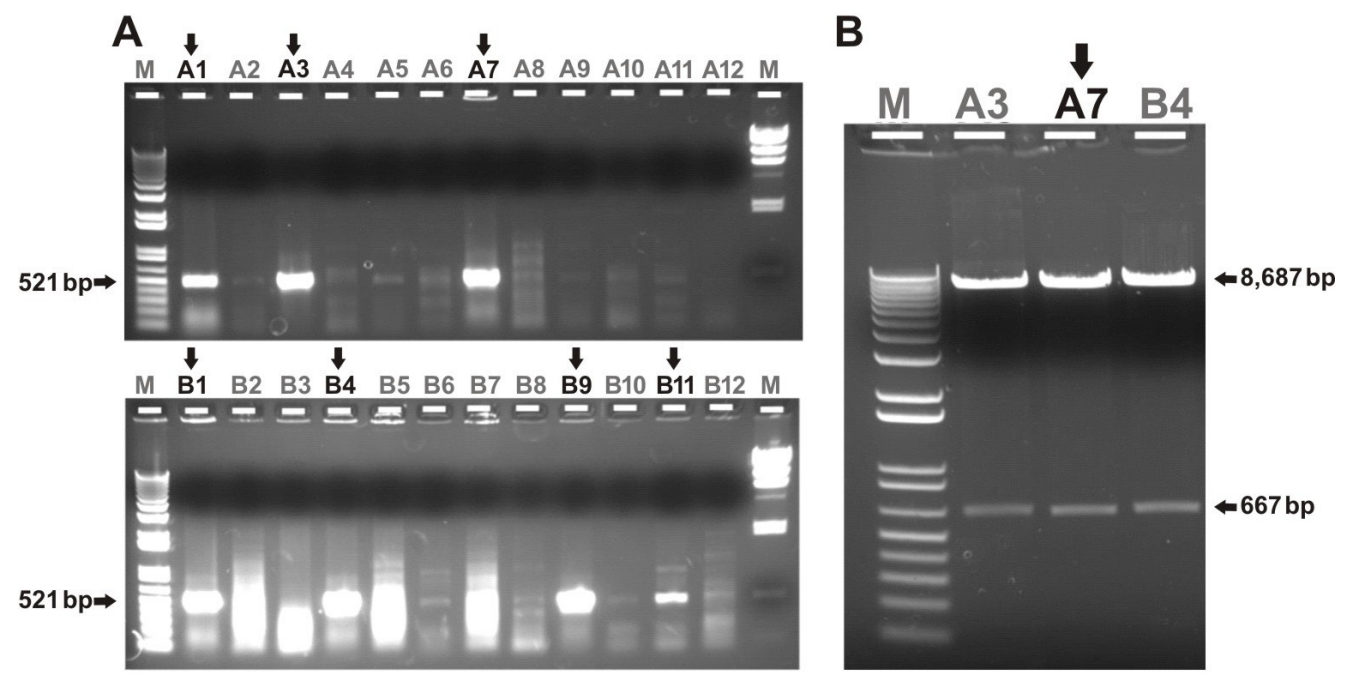

Figure 1. Cloning IRES in pMFG-P140K. A. Direct colony identification by PCR: 24 of the individual colonies (lanes $A 1$ to $A 12$ and $B 1$ to $B 12$ ) were selected randomly and cultured for $3 \mathrm{~h}$ at $37^{\circ} \mathrm{C}$ in Luria-Bertani (LB) medium (+ ampicillin). Then, some bacteria were submitted to PCR and $5 \mu \mathrm{L}$ from each PCR sample was separated electrophoretically on a 1\% agarose gel. Clones A1, A3, A7, B1, B4, B9, and B11 (indicated by arrows) have DNA products of the expected size. B. To demonstrate the presence and correct orientation of the insert, three PCRpositive clones were analyzed by restriction enzyme digestion using XhoI and NcoI. The correct orientation of insert should result in two fragments of 8,687 and $667 \mathrm{bp}$ as observed for the clones. Lanes $M=1-\mathrm{kb}$ plus ladder and Lambda DNA digested with HindIII; Lanes A3, A7 and B4 XhoI/NcoI digested positive IRES clones in pMFG-P140K.

\section{PCR cloning of cDNA FVIII-FL}

Clone A7 was used to clone FVIII-FL cDNA upstream of IRES. Figure 2A shows the results of PCR analysis from 24 randomly selected ampicillin-resistant colonies. Of the 24 colonies, 2 yielded a strong 398-bp PCR product (Figure 2A). These colonies were then analyzed with restriction enzymes (Figure $2 \mathrm{~B}$ ) and the DNA sequenced (data not shown), 
confirming the authenticity of the construct. Clone A6 denoted pMFG-FVIII-FL-I-P140K was selected for further analyses. Its structure is shown in Figure 2C.
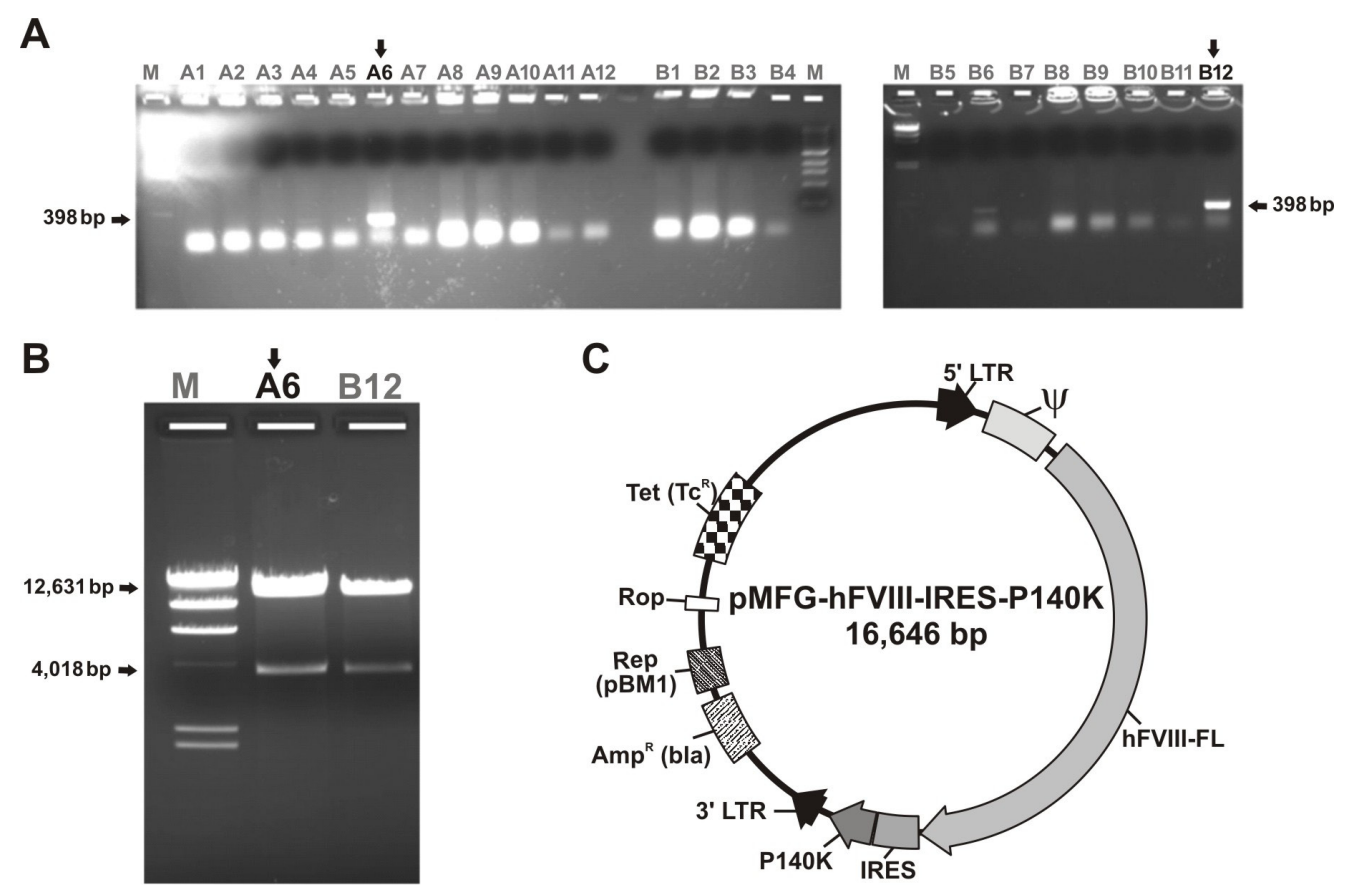

Figure 2. Cloning FVIII-FL in pMFG-I-P140K. A. Direct positive colony identification: 24 of the individual colonies (lanes $A 1$ to $A 12$ and $B 1$ to $B 12$ ) were selected randomly and cultured for $3 \mathrm{~h}$ at $37^{\circ} \mathrm{C}$ in $\mathrm{LB}$ medium (+ ampicillin). The bacteria were then submitted to PCR and $5 \mu \mathrm{L}$ from each PCR sample was separated electrophoretically on a $1 \%$ agarose gel. Two colonies showed the expected band size mobility (A6 and B12, indicated by arrows). B. To demonstrate the presence and correct orientation of the insert; two PCR-positive clones were analyzed by restriction enzyme digestion using $N c o$ I and $N s i$ I enzymes. The correct orientation of DNA insert is expected (two fragments of 12,631 and 4,018 bp as was observed for both clones). Lanes $M=$ Lambda DNA digested with HindIII and 100-bp DNA ladder; lanes $A 6$ and B12 $=$ NcoI/NsiI digested positive FVIII-FL clones in pMFG-I-P140K(A7). C. Map of the bicistronic vector pMFG-hFVIII-I-P140K(A6). Features indicated as follows: 5'LTR promoter; extended packaging signal; FVIII-FL; internal ribosome entry site from pLXIN; P140K cDNA; 3'LTR; ampicillinresistance gene ( $\beta$-lactamase); the Rep site (replicon) derived from pMB1 vector; Rop gene that regulates copy number, and the tetracycline-resistant gene $\left(\right.$ tet $\left.^{R}\right)$.

\section{PCR cloning of cDNA eGFP}

We next generated a bicistronic control vector by cloning eGFP cDNA from peGFP-C1 DNA plasmid (Clontech) upstream of IRES. Figure 3A shows PCR analysis of 24 randomly selected ampicillin-resistant colonies. Of the 26 colonies, 6 yielded a strong 643-bp PCR product. These colonies were then analyzed with restriction enzymes (Figure 3B), DNA was sequenced (data not shown), and the identity of the construct was confirmed. Clone A8 denoted pMFGeGFP-I-P140K was selected for further analyses. Its structure is shown in Figure 3C. 
A
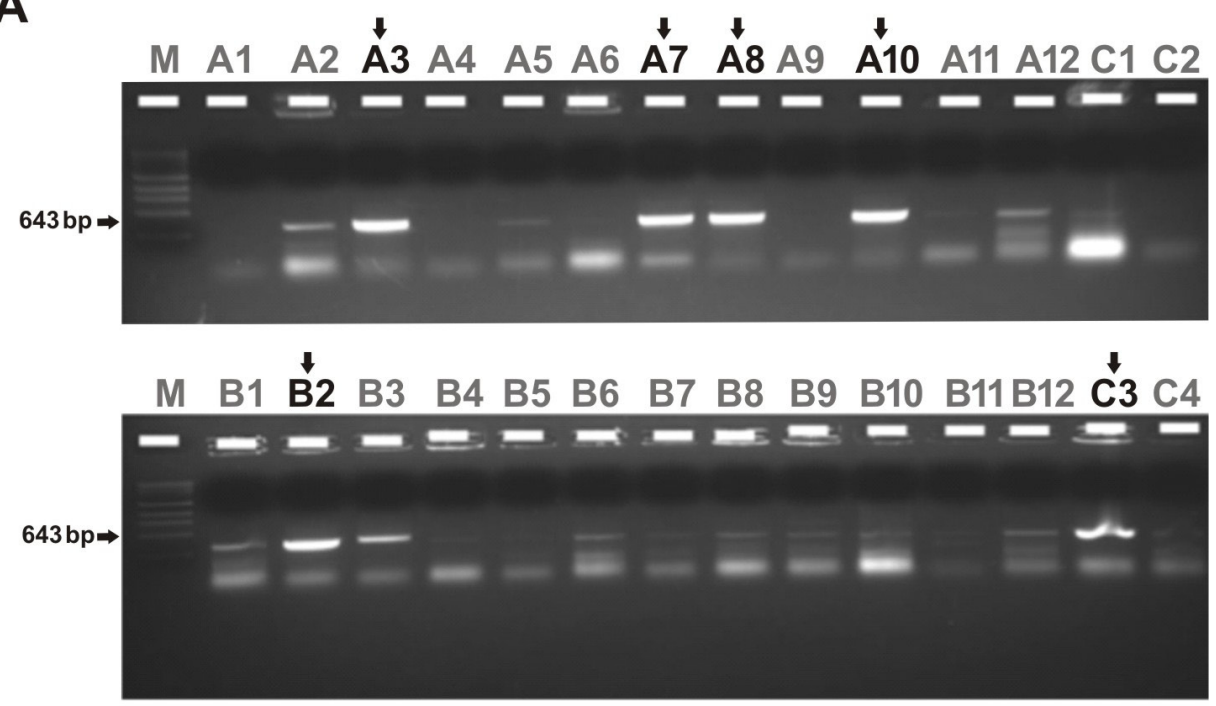

B

C

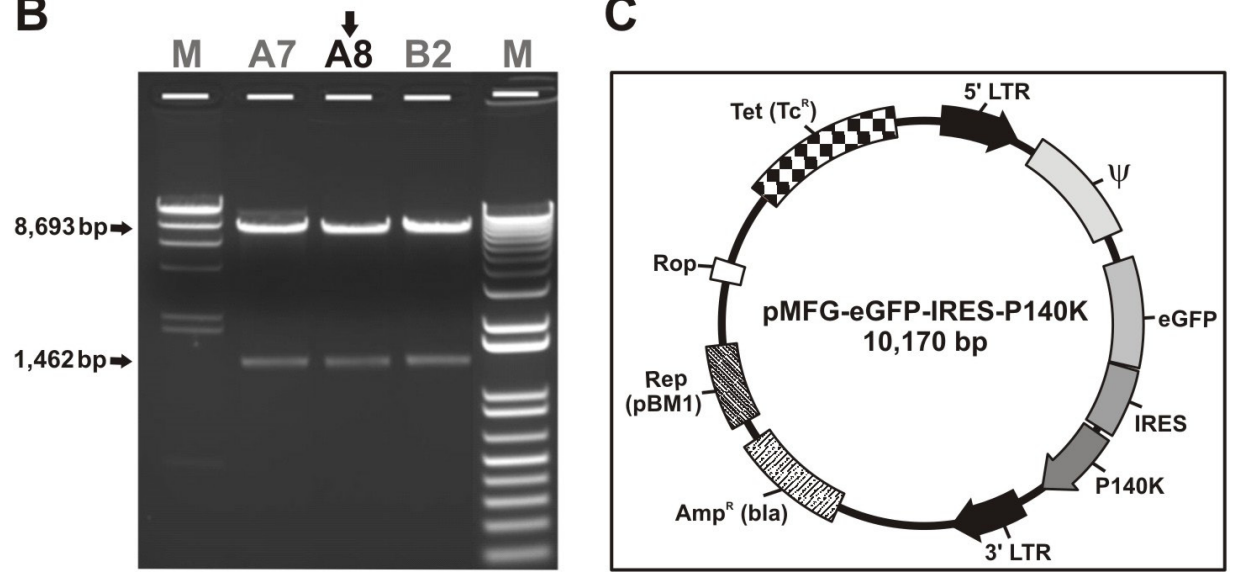

Figure 3. Cloning eGFP in pMFG-I-P140K(A7). A. Direct positive colony identification: 28 of the colonies (lanes $A 1$ to $A 12, B 1$ to $B 12$ and $C 1$ to $C 4$ ) were selected randomly and cultured for $3 \mathrm{~h}$ at $37^{\circ} \mathrm{C}$ in $\mathrm{LB}$ medium (+ ampicillin). Some bacteria were submitted to PCR and $5 \mu \mathrm{L}$ from each PCR sample was separated electrophoretically on a $1 \%$ agarose gel. Six colonies showed the expected band size (A3, A7, A8, A10, B2, and C3). B. To confirm the presence and correct orientation of the insert, six PCR-positive clones were analyzed by restriction enzyme digestion using NcoI. The correct orientation of the DNA insert should result in two fragments of 8,693 and 1,462 bp as it was observed for all clones. Lanes $M=$ Lambda DNA digested with HindIII, 100-bp DNA laldder and 1-kb DNA ladder; Lanes $A 7, A 8$ and $B 2=N c o$ I digested positive eGFP clones in pMFG-I-P140K(A7). C. Map of the bicistronic vector pMFG-eGFP-I-P140K(A8). Features indicated as in legend to Figure 2 with eGFP cDNA instead FVIII-FL cDNA.

\section{Analysis of expression level in transfected $293 \mathrm{~T}$ cells}

We next focused on analysis of the FVIII-FL, eGFP and P140K mRNA expression levels in the context of the same backbone expression vector and same cell line. We trans- 
fected 293T cells with pMFG-FVIII-FL-I-P140K and pMFG-eGFP-I-P140K simultaneously and compared the expression levels of these transgene mRNAs in 293T-FVIII-FL/P140K, 293T-eGFP/P140K and virgin cells. Total RNA from all three cell lines was isolated, and it was shown that in 293T-FVIII-FL/P140K cells, the FVIII and P140K mRNAs were present at 4,410 ( \pm 931.7$)$ - and 295,400 ( $\pm 75,769)$-fold higher than in virgin cells (Figure 4A and B). In 293T-eGFP/P140K cells, the eGFP and P140K mRNAs were present at 1,501,000 $( \pm 493,700)$ and 308,000 $( \pm 139,300)$-fold higher than in virgin cells (Figure 4C and D).
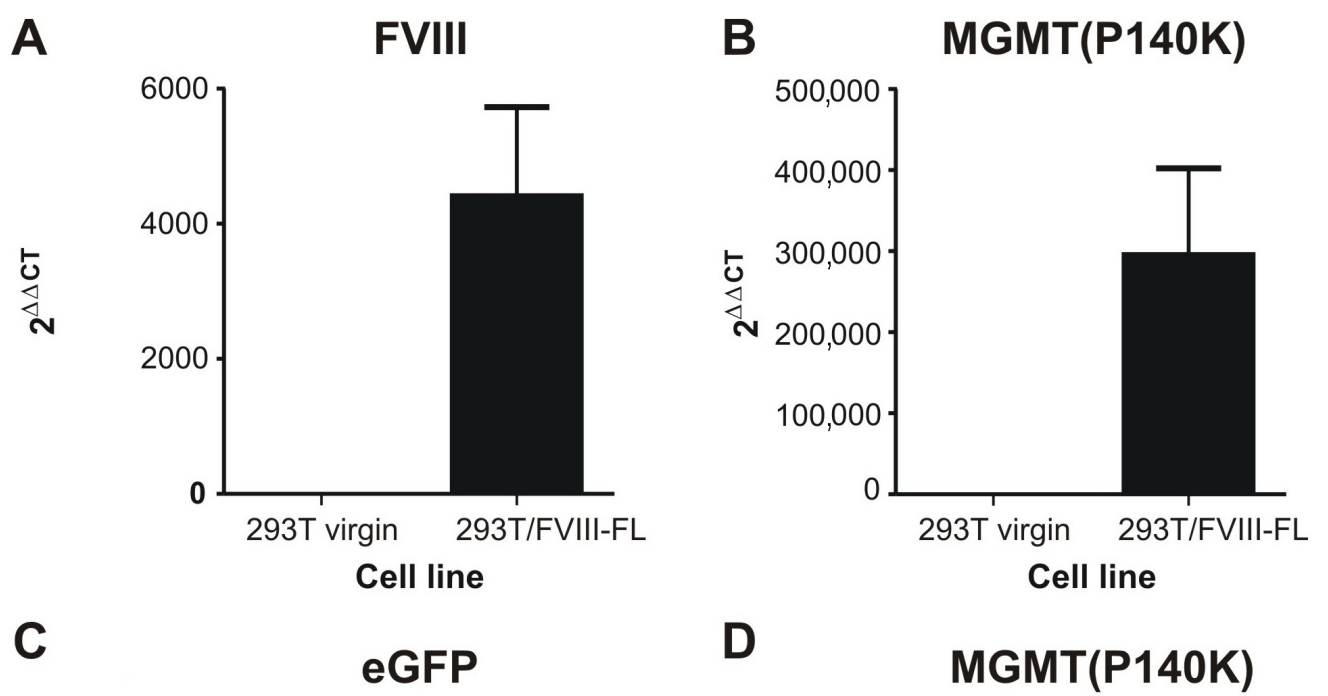

D
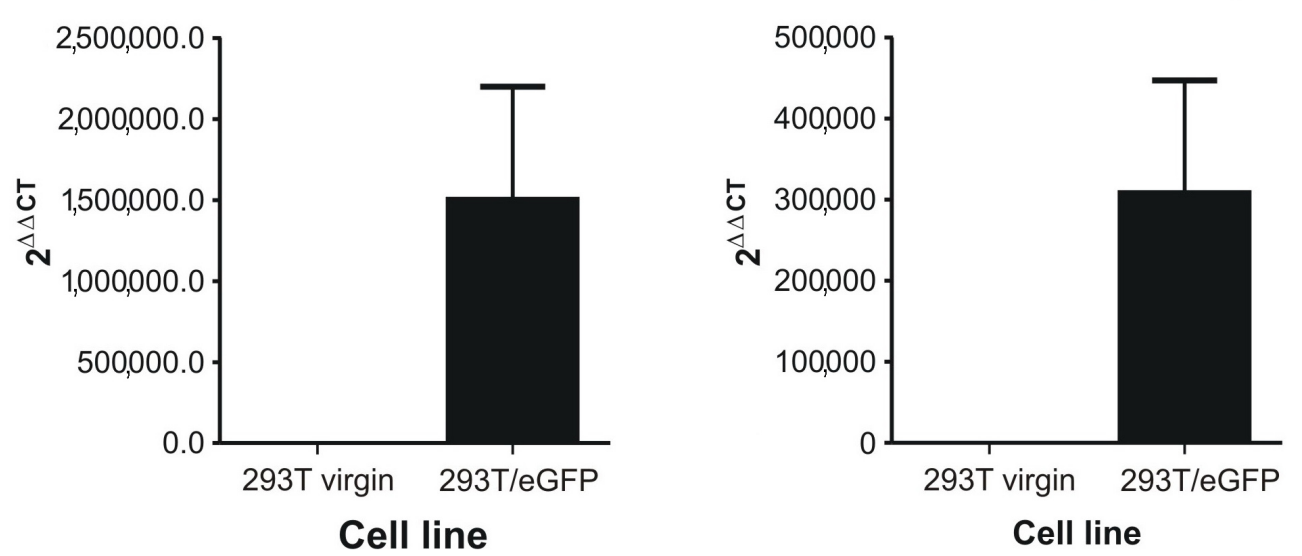

Figure 4. Expression levels of recombinant transcripts in virgin and transgenic $293 \mathrm{~T}$ cell lines. Semiquantitative RT-PCR analysis was performed using total RNA as template. The amount of FVIII, MGMT(P140K) and eGFP transcripts is relative to GAPDH.

These data showed that pMFG-hFVIII-FL-I-P140K and pMFG-eGFP-I-P140K vectors are functional bicistronic vectors and the cells express both transgenes. 


\section{FVIII activity and eGFP analysis in transfected $293 \mathrm{~T}$ cells}

To further characterize the functionality of the recombinant FVIII-FL protein, the coagulation activity of the secreted FVIII was measured using a chromogenic assay. It measured the chromophoric group pNA and was proportional to the FVIII coagulation activity in the sample. The 293T-FVIII-FL/P140K cells exhibited 0.2 IU/mL FVIII:C activity (0.19-0.22 IU/ $\mathrm{mL}) / 3 \times 10^{6}$ cells for $72 \mathrm{~h}$ cell-conditioned media after transfection.

To determine the specific activity of FVIII-FL (IU/ $\mu \mathrm{g}$ ) observed in the supernatant of 293T-FVIII-FL/P140K cells, we measured the concentration of FVIII antigen (FVIII:Ag) by ELISA. These cells exhibit FVIII concentration in the range of $45 \mathrm{ng} / \mathrm{mL}$ FVIII:Ag (42-48 ng/ $\mathrm{mL} / 3 \times 10^{6}$ cells. Therefore, the specific activity for the samples was $4.4 \mathrm{IU} / \mu \mathrm{g}$ protein and compared to $5.0 \mathrm{IU} / \mu \mathrm{g}$ by definition (see Quantification of FVIII-FL). The activity and concentration data demonstrated that the recombinant FVIII molecule secreted by 293T-FVIIIFL/P140K cells into the culture medium was biologically active.

Next, we analyzed the eGFP expression in 293T-eGFP/P140K cell line by flow cytometry and fluorescence microscopy to quantify the reporter gene expression containing the same retroviral vector backbone but instead coding for eGFP. Fluorescence microscopic analysis allowed monitoring the green fluorescence cells in culture (Figure 5A). Flow analysis showed about $15-17 \%$ of transfected $293 \mathrm{~T}$ cells expressing eGFP fluorescence $72 \mathrm{~h}$ after transfection (Figure 5B). These results confirmed that the pMFG-eGFP-I-P140K reporter system allows visualization and diagnostic of eGFP expression in vitro.
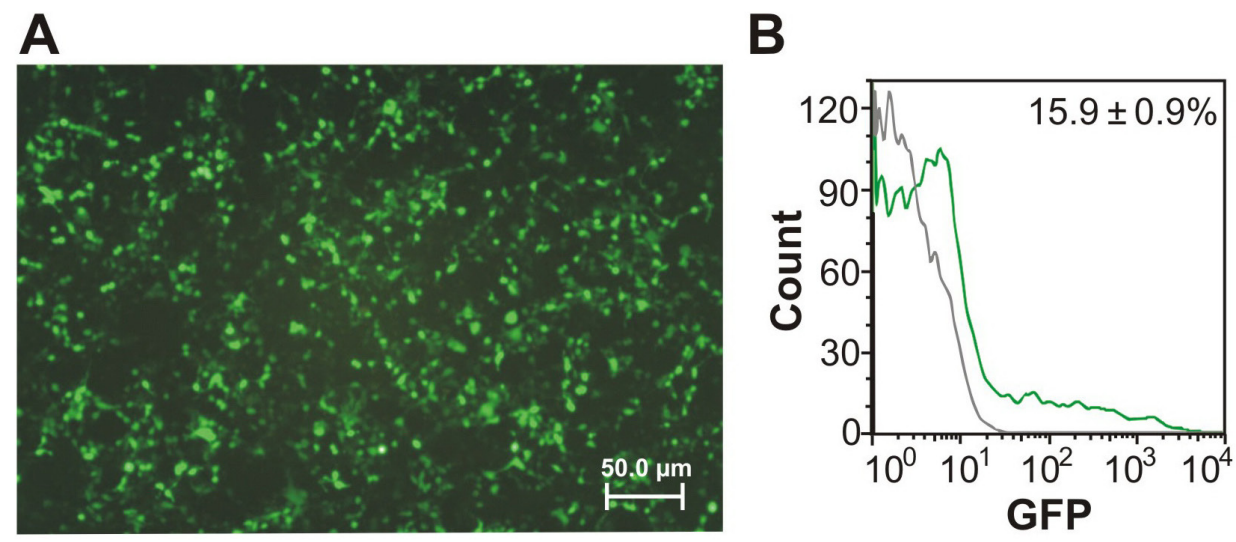

Figure 5. Expression of eGFP in 293T-eGFP/P140K cells. A. Characterization of the 293T-FVIII-FL/eGFP culture: representative fluorescence microphotograph. Note the presence of fluorescent cells in 293T cells modified with pMFG-eGFP-I-P140K. Scale bar corresponds to $50 \mu \mathrm{m}$. B. Histograms showing the number of cells (count) $v s$ the fluorescence exhibited level, green fluorescent protein (GFP). The peak outlined in green represents cells that were expressing GFP.

Therefore, both FVIII-FL and eGFP proteins expressed from the retroviral vector described here were functional. Also, these vectors allowed the expression of similar levels of MGMT(P140K), which can be used for further in vitro assays to select a human cell line with high amount of recombinant proteins. 


\section{DISCUSSION}

Most recombinant therapeutic proteins available are produced in nonhuman mammalian cell lines and provoke some immune response (Dingermann, 2008). Therefore, human cell line-derived recombinant products can potentially produce clinical benefits and have been considered by Octapharma, since two years ago, when the first clinical trial was started using one recombinant B domain deleted-FVIII produced in a human cell line. However, based on previous studies that demonstrated the role of FVIII-B domain in its intracellular processing and transport (Pipe, 2009), the development of the FVIII-FL molecule should improve some outcomes in replacement or gene therapy trials.

In the present study, we designed a gamma-retroviral vector for the efficient delivery of FVIII-FL to a human cell line for transient gene expression. We developed bicistronic vectors from MFG-based retroviral vectors by introducing an IRES element to genetically link a therapeutic gene with a drug-resistance element.

Our results show the FVIII-FL and eGFP mRNAs transcribed from MoMuLV LTR promoter in 293T-FVIII-FL/P140K cells and 293T-eGFP/P140K cells are approximately 4000and 1,500,000-fold higher compared to mRNAs of virgin cells, respectively. The intrinsic properties of FVIII-FL mRNA, which contribute to the reduced accumulation of FVIII-FL mRNA in several cell lines (Pipe, 2009), can explain the difference in the expression of FVIII-FL and eGFP. Also, it has been demonstrated that the FVIII-FL cDNA contains a 305-bp motif, which functions as a transcriptional repressor element (Fallaux et al., 1996). These cited investigators showed that these transcription inhibitor sequences of FVIII-FL cDNA expression colocalize with the sequences that mediate the stimulatory effect of butyrate. Therefore, further studies involving the treatment of 293T-FVIII-FL/P140K cell line with butyrate should be performed to determine whether or not this cell line will respond to this treatment and whether or not it will be possible to increase the amount of the FVIII protein produced. Furthermore, we compared the resistance gene in both bicistronic vectors, which was cloned downstream of IRES. We found that the MGMT(P140K) cDNA was expressed at similar levels in both $293 \mathrm{~T}$ cell lines. As expected, since the use of IRES elements is an alternative strategy for eukaryotic cells for initiating protein translation beyond the canonical cap-dependent ribosome binding (Pestova et al., 2001), it should not interfere with the level of transcribed mRNA (Wang et al., 2005).

Despite the low level of FVIII-FL expression compared to MGMT(P140K) from the pMFG-FVIII-FL/P140K vector, we consistently obtained protein production in the 293TFVIII-FL/P140K cell line. Analyses of the FVIII antigen concentration by immunoassays revealed that there was good correlation between the FVIII concentration and the FVIII activity (data not shown). In this study, we observed that the FVIII protein concentrations in culture medium varied from 0.19 to $0.22(\mathrm{IU} / \mathrm{mL}) / 3 \times 10^{6}$ cells within $72 \mathrm{~h}$ and corresponded to 4.4 $\mathrm{U} / \mu \mathrm{g}$ compared to $5.0 \mathrm{U} / \mu \mathrm{g}$, which is the standard definition of the specific activity of FVIII in plasma.

Considering that the bicistronic vector generated here also expressed a P140K mutant methyltransferase (MGMT) to select gene-modified cells by adding $\mathrm{O}^{6}-\mathrm{BG} / \mathrm{O}^{6}$-alkylating drug combinations, further assays can be performed to increase the amount of the recombinant FVIII-FL protein, and the eGFP vector can be used as a control to establish the optimum drug regimen for in vitro production. To date, we have demonstrated that this system is suitable for an in vitro selection strategy (Reese et al., 1996; Zielske et al., 2003; Fontes et al., 2006). 
Pre-clinical studies have shown efficient stable in vivo selection of genetically modified hematopoietic cells in mice (Zielske et al., 2003), dogs (Neff et al., 2003, 2005) and nonhuman primates (Larochelle et al., 2006; Beard et al., 2010) and also for monogenic diseases such hemophilia B (Chang et al., 2008) and $\beta$-thalassemia (Persons et al., 2003), using viral vector to transfer an MGMT drug-resistance gene into bone marrow cells. Therefore, further studies in mice or in a large animal model will ultimately determine the feasibility of using the MGMT selection system with the vector generated here in the context of gene and cell therapy for hemophilia A disease.

Human cultured cell-based production is an attractive alternative manufacturing platform for biopharmaceuticals. The 293T cell line technology platform offers many advantages for large-scale protein production over traditional biomanufacturing methods due to its high transfectability, which allows the production of numerous recombinant proteins. In the present study, the use of the 293T cell line and the construct described here can easily be used for other proteins. Also, we explored the advantage of using a vector which expresses MGMT(P140K) as a marker gene, which has not been previously demonstrated for hemophilia A. We showed that the MGMT(P140K) gene is expressed at similar levels by both vectors indicating that both bicistronic vectors are functional. The transfection of constructs containing the FVIII-FL gene and MGMT into cultured cells results in recombinant protein production within a short time and may also be useful for generating a human cell line with higher amount of FVIII-FL and also either for gene and cell therapy pre-clinical models or to highlight the importance of B-domain FVIII for hemophilia A research.

\section{ACKNOWLEDGMENTS}

Research supported by FINEP(Grant\#01.07.0652.00), FAPESP(Grant\#1998/142476) and CNPq (Grant \#314458/2009-3 and \#2008/57877-3). We thank Sandra Navarro Bresciani for preparing the figures.

\section{Conflict of interest statement}

The authors declare that they have no conflicts of interest relevant to this manuscript submitted to Genetics and Molecular Research.

\section{REFERENCES}

Ashrani AA, Reding MT, Shet A, Osip J, et al. (2004). Successful liver transplantation in a patient with severe haemophilia A and a high-titre factor VIII inhibitor. Haemophilia 10: 735-737.

Beard BC, Trobridge GD, Ironside C, McCune JS, et al. (2010). Efficient and stable MGMT-mediated selection of longterm repopulating stem cells in nonhuman primates. J. Clin. Invest. 120: 2345-2354.

Becker S, Simpson JC, Pepperkok R, Heinz S, et al. (2004). Confocal microscopy analysis of native, full length and B-domain deleted coagulation factor VIII trafficking in mammalian cells. Thromb. Haemost. 92: 23-35.

Bovenschen N, Rijken DC, Havekes LM, van Vlijmen BJ, et al. (2005). The B domain of coagulation factor VIII interacts with the asialoglycoprotein receptor. J. Thromb. Haemost. 3: 1257-1265.

Camire RM (2010). Hemophilia: basic and translational science. Expert. Rev. Hematol. 3: 149-151.

Chang AH, Stephan MT, Lisowski L and Sadelain M (2008). Erythroid-specific human factor IX delivery from in vivo selected hematopoietic stem cells following nonmyeloablative conditioning in hemophilia B mice. Mol. Ther. 16: $1745-1752$. 
Cohen SN, Chang AC and Hsu L (1972). Nonchromosomal antibiotic resistance in bacteria: genetic transformation of Escherichia coli by R-factor DNA. Proc. Natl. Acad. Sci. U. S. A. 69: 2110-2114.

Dalby B, Cates S, Harris A, Ohki EC, et al. (2004). Advanced transfection with Lipofectamine 2000 reagent: primary neurons, siRNA, and high-throughput applications. Methods 33: 95-103.

Dingermann T (2008). Recombinant therapeutic proteins: production platforms and challenges. Biotechnol. J. 3: 90-97.

Fallaux FJ, Hoeben RC, Cramer SJ, van den Wollenberg DJ, et al. (1996). The human clotting factor VIII cDNA contains an autonomously replicating sequence consensus- and matrix attachment region-like sequence that binds a nuclear factor, represses heterologous gene expression, and mediates the transcriptional effects of sodium butyrate. Mol. Cell Biol. 16: 4264-4272.

Fay PJ (1993). Factor VIII structure and function. Thromb. Haemost. 70: 63-67.

Fontes AM, Davis BM, Encell LP, Lingas K, et al. (2006). Differential competitive resistance to methylating versus chloroethylating agents among five O6-alkylguanine DNA alkyltransferases in human hematopoietic cells. Mol. Cancer Ther. 5: 121-128.

Franchini M (2010). Plasma-derived versus recombinant factor VIII concentrates for the treatment of haemophilia A: recombinant is better. Blood Transfus. 8: 292-296.

Furie B and Furie BC (1988). The molecular basis of blood coagulation. Cell 53: 505-518.

Goudemand J, Rothschild C, Demiguel V, Vinciguerrat C, et al. (2006). Influence of the type of factor VIII concentrate on the incidence of factor VIII inhibitors in previously untreated patients with severe hemophilia A. Blood 107: 46-51.

Grillberger L, Kreil TR, Nasr S and Reiter M (2009). Emerging trends in plasma-free manufacturing of recombinant protein therapeutics expressed in mammalian cells. Biotechnol. J. 4: 186-201.

Gringeri A, Mantovani LG, Scalone L and Mannucci PM (2003). Cost of care and quality of life for patients with hemophilia complicated by inhibitors: the COCIS Study Group. Blood 102: 2358-2363.

Josephson CD and Abshire T (2004). The new albumin-free recombinant factor VIII concentrates for treatment of hemophilia: do they represent an actual incremental improvement? Clin. Adv. Hematol. Oncol. 2: 441-446.

Larochelle A, Krouse A, Metzger M, Orlic D, et al. (2006). AMD3100 mobilizes hematopoietic stem cells with long-term repopulating capacity in nonhuman primates. Blood 107: 3772-3778.

Manco-Johnson MJ (2003). Update on treatment regimens: prophylaxis versus on-demand therapy. Semin. Hematol. 40: 3-9.

Neff T, Horn PA, Peterson LJ, Thomasson BM, et al. (2003). Methylguanine methyltransferase-mediated in vivo selection and chemoprotection of allogeneic stem cells in a large-animal model. J. Clin. Invest. 112: 1581-1588.

Neff T, Beard BC, Peterson LJ, Anandakumar P, et al. (2005). Polyclonal chemoprotection against temozolomide in a large-animal model of drug resistance gene therapy. Blood 105: 997-1002.

Persons DA, Allay ER, Sawai N, Hargrove PW, et al. (2003). Successful treatment of murine beta-thalassemia using in vivo selection of genetically modified, drug-resistant hematopoietic stem cells. Blood 102: 506-513.

Pestova TV, Kolupaeva VG, Lomakin IB, Pilipenko EV, et al. (2001). Molecular mechanisms of translation initiation in eukaryotes. Proc. Natl. Acad. Sci. U. S. A. 98: 7029-7036.

Pfaffl MW (2001). A new mathematical model for relative quantification in real-time RT-PCR. Nucleic Acids Res. 29: e45.

Pipe SW (2008). Recombinant clotting factors. Thromb. Haemost. 99: 840-850.

Pipe SW (2009). Functional roles of the factor VIII B domain. Haemophilia 15: 1187-1196.

Pipe SW, High KA, Ohashi K, Ural AU, et al. (2008). Progress in the molecular biology of inherited bleeding disorders. Haemophilia 14 (Suppl 3): 130-137.

Pipe SW, Miao H, Butler SP, Calcaterra J, et al. (2011). Functional factor VIII made with von Willebrand factor at high levels in transgenic milk. J. Thromb. Haemost. 9: 2235-2242.

Pittman DD, Alderman EM, Tomkinson KN, Wang JH, et al. (1993). Biochemical, immunological, and in vivo functional characterization of B-domain-deleted factor VIII. Blood 81: 2925-2935.

Reese JS, Koc ON, Lee KM, Liu L, et al. (1996). Retroviral transduction of a mutant methylguanine DNA methyltransferase gene into human CD34 cells confers resistance to O6-benzylguanine plus 1,3-bis(2-chloroethyl)-1-nitrosourea. Proc. Natl. Acad. Sci. U. S. A. 93: 14088-14093.

Soucie JM, Evatt B and Jackson D (1998). Occurrence of hemophilia in the United States. The Hemophilia Surveillance System Project Investigators. Am. J. Hematol. 59: 288-294.

Toole JJ, Pittman DD, Orr EC, Murtha P, et al. (1986). A large region (approximately equal to $95 \mathrm{kDa}$ ) of human factor VIII is dispensable for in vitro procoagulant activity. Proc. Natl. Acad. Sci. U. S. A. 83: 5939-5942.

Van Damme A, Thorrez L, Ma L, Vandenburgh H, et al. (2006). Efficient lentiviral transduction and improved engraftment of human bone marrow mesenchymal cells. Stem Cells 24: 896-907.

Wang H, Shen XT, Ye R, Lan SY, et al. (2005). Roles of the polypyrimidine tract and 3' noncoding region of hepatitis C virus RNA in the internal ribosome entry site-mediated translation. Arch. Virol. 150: 1085-1099. 
Yokoyama S, Bartlett A, Dar FS, Heneghan M, et al. (2011). Outcome of liver transplantation for haemophilia. HPB 13: 40-45.

Zhao H, Pestina TI, Nasimuzzaman M, Mehta P, et al. (2009). Amelioration of murine beta-thalassemia through drug selection of hematopoietic stem cells transduced with a lentiviral vector encoding both gamma-globin and the MGMT drug-resistance gene. Blood 113: 5747-5756.

Zielske SP, Reese JS, Lingas KT, Donze JR, et al. (2003). In vivo selection of MGMT(P140K) lentivirus-transduced human NOD/SCID repopulating cells without pretransplant irradiation conditioning. J. Clin. Invest. 112: 1561-1570. 